PROCEEDINGS OF THE

AMERICAN MATHEMATICAL SOCIETY

Volume 138, Number 3, March 2010, Pages 907-919

S 0002-9939(09)09984-5

Article electronically published on November 5, 2009

\title{
AUTOMATIC CONTINUITY VIA ANALYTIC THINNING
}

\author{
N. H. BINGHAM AND A. J. OSTASZEWSKI \\ (Communicated by Michael T. Lacey) \\ To Roy Davies on the occasion of his 80th birthday
}

\begin{abstract}
We use Choquet's analytic capacitability theorem and the Kestelman-Borwein-Ditor theorem (on the inclusion of null sequences by translation) to derive results on 'analytic automaticity' - for instance, a stronger common generalization of the Jones/Kominek theorems that an additive function whose restriction is continuous/bounded on an analytic set $T$ spanning $\mathbb{R}$ (e.g., containing a Hamel basis) is continuous on $\mathbb{R}$. We obtain results on 'compact spannability' - the ability of compact sets to span $\mathbb{R}$. From this, we derive Jones' Theorem from Kominek's. We cite several applications, including the Uniform Convergence Theorem of regular variation.
\end{abstract}

\section{INTRODUCTION}

This paper, on additive functions, is a sequel to BOst, where we study subadditive functions, and in turn leads on to the companion paper BOst6, on convex and related functions.

Darboux's theorem of 1875 ([Dar, [AD, Section 21.6]) asserts that for additive functions, local boundedness implies continuity. Ostrowski's result of 1929 Ostr that a (mid-point) convex and so a fortiori an additive function bounded above on some set $T$ of positive measure is continuous may be regarded as a thinning out of Darboux's assumed 'local' character from a property holding on an interval to the same property holding only on a set of positive measure. From this perspective Jones' theorem of 1942 Jones that an additive function continuous on a set $T$ which is analytic (for definition and background see Rog2]) and contains a Hamel basis is continuous may be seen as a further thinning out.

There is a closely related, more recent, result of Z. Kominek in 1981 Kom2, which says that an additive function bounded on a set $T$ which is analytic and contains a Hamel basis is continuous. As boundedness is here limited to $T$, it is not immediately clear what the logical relationship between these theorems is, despite the almost identical proof structure, as elegantly derived by Kominek (from ideas which he attributes as being implicitly in Jones). For convenience, let us say briefly that $T$ is a spanning set when $\mathbb{R}$ regarded as a vector space over $\mathbb{Q}$ has $T$ as a spanning set of vectors. (In the presence of the Axiom of Choice, AC, a spanning

Received by the editors June 28, 2008, and, in revised form, April 3, 2009.

2000 Mathematics Subject Classification. Primary 26A03.

Key words and phrases. Jones' theorem, Kominek's theorem, analytic set, Choquet capacity, Hamel basis, uniform convergence theorem, regular variation, automatic continuity. 
set contains a Hamel basis; as is well-known, AC implies that every vector space has a basis. We note in passing that the converse is also true; see [Bl].)

The theorems of Jones and Kominek are results on automatic continuity (see [THJ] for background and references) of a particular type: the set $T$, on which a property is assumed, is analytic (and 'big enough' - say, spanning a subset of positive measure or a co-meagre set and hence spanning all of $\mathbb{R}$, or containing a Hamel basis if the Axiom of Choice is assumed). Note that such 'big enough' sets may themselves be small - for example, the Cantor set $C$ is compact (hence analytic) and both null and meagre; but $C+C=[0,2]$ (see e.g. Fal] p. 108, Kucz] p. 50), as $C$ is the subset of reals in $[0,1]$ containing only 0 and 2 in their ternary expansions, and $\{0,2\}$ generates $\mathbb{Z}_{3}$. Thus $C$ spans $\mathbb{R}$.

We unify results of the Jones-Kominek type, which we dub theorems on analytic automaticity, by showing that both are instances of a stronger theorem of the same type. See [BOst6] for a range of such theorems touching convex functions (see Note 1 at the end of the paper) and making the connection with the uniform convergence theorem of regular variation (for which see e.g. [BGT]).

Our theorem identifies circumstances under which a weak property, such as local boundedness, that has been given 'analytic thinning out' still implies a strong property, such as continuity. The theorem calls for three ingredients: an initial 'weak implies strong' hypothesis (for which the canonical example is Darboux's theorem), the sequential character of the weak property (to be defined), and a modicum of vector-space structure (given by the theorem, but see also the re-formulation in Section 5 that makes explicit the underlying sequential combinatorics, which we require for BOst6] ).

Definitions. For a family $\mathcal{F}$ of functions from $\mathbb{R}^{d}$ to $\mathbb{R}$, we denote by $\mathcal{F}(T)$ the family $\{f \mid T: f \in \mathcal{F}\}$ of functions in $\mathcal{F}$ restricted to $T \subseteq \mathbb{R}^{d}$. Let us denote a convergent sequence with limit $\mathbf{x}_{0}$ by $\left\{\mathbf{x}_{n}\right\} \rightarrow \mathbf{x}_{0}$. We say that the property $\mathcal{Q}$ of functions (with property being regarded set-theoretically, i.e. as a family of functions from $\mathbb{R}^{d}$ to $\left.\mathbb{R}\right)$ is sequential on $T$ if

$f \in \mathcal{Q}$ iff $\left(\forall\left\{\mathbf{x}_{n}: n>0\right\} \subseteq T\right)\left[\left(\left\{\mathbf{x}_{n}\right\} \rightarrow \mathbf{x}_{0}\right) \Longrightarrow f \mid\left\{\mathbf{x}_{n}: n>0\right\} \in \mathcal{Q}\left(\left\{\mathbf{x}_{n}: n>0\right\}\right)\right]$.

If we further require the limit point to be enumerated in the sequence, we call $\mathcal{Q}$ completely sequential on $T$ if

$$
f \in \mathcal{Q} \text { iff }\left(\forall\left\{\mathbf{x}_{n}\right\} \subseteq T\right)\left[\left(\left\{\mathbf{x}_{n}\right\} \rightarrow \mathbf{x}_{0}\right) \Longrightarrow f \mid\left\{\mathbf{x}_{n}\right\} \in \mathcal{Q}\left(\left\{\mathbf{x}_{n}\right\}\right)\right] .
$$

It is thus useful to distinguish between $\omega=\{0,1,2, \ldots\}$ and $\mathbb{N}=\{1,2,3, .$.$\} . Our$ interest rests on properties that are completely sequential; our theorem below contains a condition referring to completely sequential properties, that is, the condition is required to hold on convergent sequences with limit included (and so on a compact set) rather than on arbitrary sequences.

Note that if $\mathcal{Q}$ is (completely) sequential, then $f \mid\left\{\mathbf{x}_{n}\right\} \in \mathcal{Q}\left(\left\{\mathbf{x}_{n}\right\}\right)$ iff $f \mid\left\{\mathbf{x}_{n}: n \in\right.$ $\mathbb{M}\} \in \mathcal{Q}\left(\left\{\mathbf{x}_{n}: n \in \mathbb{M}\right\}\right)$, for every infinite $\mathbb{M}$. The theorem below gives conditions for the following analytic thinning principle to hold in $\mathcal{F}$ : if

$$
\mathcal{Q}\left(\mathbb{R}^{d}\right) \Longrightarrow \mathcal{P}\left(\mathbb{R}^{d}\right)
$$

holds, then

holds for analytic $T$.

$$
\mathcal{Q}(T) \Longrightarrow \mathcal{P}\left(\mathbb{R}^{d}\right)
$$


Main Theorem (Analytic Automaticity Theorem). Suppose that

(a) functions of $\mathcal{F}$ having the property $\mathcal{Q}$ on $\mathbb{R}^{d}$ have property $\mathcal{P}$ on $\mathbb{R}^{d}$, where $\mathcal{Q}$ is a property of functions from $\mathbb{R}^{d}$ to $\mathbb{R}$ that is completely sequential on $\mathbb{R}^{d}$;

(b) $\mathcal{F}$ preserves $\mathcal{Q}$ under vector addition and subtraction on compact sets and also under shift, that is:

(i) for compact sets $S$ and $T$, functions of $\mathcal{F}$ having $\mathcal{Q}$ on $S$ and $T$ have $\mathcal{Q}$ on $S \pm T$

(ii) functions of $\mathcal{F}$ having $\mathcal{Q}$ on any $T \subseteq \mathbb{R}^{d}$ have $\mathcal{Q}$ on $\tau+T:=\{\tau+t: t \in$ $T\}$, for any $\tau \in \mathbb{R}^{d}$.

Then, for any analytic set $T$ spanning $\mathbb{R}^{d}$ as a vector space over $\mathbb{Q}$ (e.g. containing a Hamel basis), functions of $\mathcal{F}$ having $\mathcal{Q}$ on $T$ have $\mathcal{P}$ on $\mathbb{R}^{d}$.

Remark. In applications, as in the two examples that follow, the conditions (i) and (ii) need only be verified on compact sets arising as convergent sequences with limit points included, in view of the properties needing to be completely sequential. This is indeed the form that is relevant in examples, but we have not included an extra assertion here along these lines to avoid overburdening the statement of the theorem. See, however, Section 5.

Example 1. The class of additive functions, $\mathcal{A d d}$, preserves $\mathcal{C}$, the continuous functions, under vector sums and differences on compact domains; i.e. for $f \in \mathcal{A} d d$ and $S, T$ compact, if $f \mid S \in \mathcal{C}(S)$ and $f \mid T \in \mathcal{C}(T)$, then $f \mid S \pm T \in \mathcal{C}(S \pm T)$. Indeed let $u_{n}=s_{n} \pm t_{n} \in S \pm T$. Then $\left\{s_{n}: n \in \omega\right\}$ and $\left\{t_{n}: n \in \omega\right\}$ are precompact sets. By compactness of $S$ and $T$, without loss of generality we may assume that $s_{n} \rightarrow s \in S$ and $t_{n} \rightarrow t \in T$. Then, by additivity, $\lim f\left(s_{n} \pm t_{n}\right)=\lim \left[f\left(s_{n}\right) \pm f\left(t_{n}\right)\right]$, and by continuity $\lim \left[f\left(s_{n}\right) \pm f\left(t_{n}\right)\right]=f(s) \pm f(t)$. Thus $f$ is continuous on $S \pm T$.

Example 2. The class of additive functions, $\mathcal{A} d d$, preserves $\mathcal{B}_{\text {loc }}$, the locally bounded functions, under vector sums and differences on compact domains; i.e. for $f \in \mathcal{A} d d$ and $S, T$ compact, if $f \mid S \in \mathcal{B}_{\text {loc }}(S)$ and $f \mid T \in \mathcal{B}_{\text {loc }}(T)$, then $f \mid S \pm T \in$ $\mathcal{B}_{\text {loc }}(S \pm T)$. The proof is similar to but simpler than that above.

Corollary (Theorems of Jones and Kominek). Let $f$ be additive and have either a continuous restriction or a bounded restriction, $f \mid T$, where $T$ is some analytic set spanning $\mathbb{R}$. Then $f$ is continuous.

Proof. Applying the Main Theorem, let $\mathcal{F}$ be $\mathcal{A} d d$, the family of additive functions, and let $\mathcal{P}$ be $\mathcal{C}$, the family of continuous functions. Thus with $\mathcal{Q}=\mathcal{C}$ we obtain Jones' Theorem (by Example 1), and with $\mathcal{Q}=\mathcal{B}_{\text {loc }}$, the locally bounded functions, we obtain (by Example 2) a sharpened form of Kominek's Theorem (and with $\mathcal{Q}=\mathcal{B}$, the bounded functions, its original form).

The use of spanning sets here is natural, since an additive function is specified by its values on a spanning set. This idea can be made precise. Kominek (Kom2, Th. 1]) also shows that nothing less than a spanning set will do here. This - 'Kominek's other theorem' - may be regarded as a converse to the Kominek theorem above.

We will need the following result, due in the measure case in this form to Borwein and Ditor BoDi, but already known much earlier albeit in somewhat weaker form by Kestelman ([Kes, Th. 3]) and rediscovered by Trautner Trau. Much more is true; see BOst6], BOst9, BOst11]. Following J.-P. Kahane [Kah], the term 'quasi all' below refers to 'all off some meagre set'. 
Theorem (Kestelman-Borwein-Ditor Theorem). Let $\left\{z_{n}\right\} \rightarrow 0$ be a null sequence of reals. If $T$ is measurable and non-null/Baire non-meagre, then for almost all/ quasi all $t \in T$ there is an infinite set $\mathbb{M}_{t}$ such that

$$
\left\{t+z_{m}: m \in \mathbb{M}_{t}\right\} \subseteq T .
$$

\section{Some lemmas: ExPANSION AND CONTRACTION}

For clarity's sake we work in $\mathbb{R}$ rather than in $\mathbb{R}^{d}$. We begin with the common proof of the Jones and Kominek theorems as it is short, illuminating and depends on three simple lemmas, the second and third of which we need elsewhere (for the Souslin operation therein, see $(\overline{R o g} 2)$. The stronger result that Jones' Theorem implies Kominek's Theorem is deduced in the subsequent section on spannability.

Analytic Covering Lemma ([Kucz, p. 227]; cf. [Jones, Th. 11]). Let $T$ be analytic and let $f: \mathbb{R} \rightarrow \mathbb{R}$ have continuous restriction $f \mid T$. Then $T$ is covered by a countable family of bounded analytic sets on each of which $f$ is bounded.

Proof. For $k \in \omega$ define $T_{k}:=\{x \in T:|f(x)|<k\} \cap(-k, k)$. Now $\{x \in T:|f(x)|<$ $k\}$ is relatively open and so takes the form $T \cap U_{k}$ for some open subset $U_{k}$ of $\mathbb{R}$, giving the result since $U_{k}$ is analytic.

Analytic Dichotomy Lemma (Spanning). Suppose that an analytic set $T \subseteq \mathbb{R}$ spans a set of positive measure or a non-meagre set. Then $T$ spans $\mathbb{R}$.

Proof. If $T$ spans $P \subseteq \mathbb{R}$, then

$$
P \subseteq S:=\bigcup_{m, h \in \omega, m>0} \bigcup_{\mathbf{r} \in \mathbb{Z}^{h}}\left(\frac{r_{1}}{m} T+\ldots+\frac{r_{h}}{m} T\right) .
$$

In the measure case, if $P$ is non-null, it follows that for some $h, m \in \mathbb{N}$ and $\mathbf{r} \in \mathbb{Z}^{h}$, the set

$$
\frac{r_{1}}{m} T+\ldots+\frac{r_{h}}{m} T
$$

has positive measure, and hence so does $S^{\prime}=r_{1} T+\ldots+r_{h} T$. By Steinhaus' Theorem ([St], BGT, Th. 1.1.1], BOst3]), $S^{\prime}-S^{\prime}$ contains an interval around the origin, and so $T$ spans an interval, say $(-1 / k, 1 / k)$ for some $k \in \mathbb{N}$. Hence $T$ spans $(-n / k, n / k)$ for any $n \in \mathbb{N}$; i.e. $T$ spans $\mathbb{R}$.

In the category case, $S^{\prime}$ is non-meagre, and so, by the Pettis-Piccard Theorem ([Pic1], Pic2], Pet1], [BGT, Th. 1.1.1], BOst3]), $S^{\prime}-S^{\prime}$ contains an interval around the origin, hence the similar result.

In the category case, the result may also be derived from the Banach-Kuratowski Dichotomy Theorem ([Ban, Satz 1], [Kur1, Ch. VI. 13. XII], Kel, Ch. 6, Prob. P, p. 211]) by considering $S$, the subgroup generated by $T$; since $T$ is analytic, $S$ is Baire and, being non-meagre, is clopen and hence equal to all of $\mathbb{R}$, as the latter is a connected group.

In what follows, for $\alpha$ a sequence in $\omega^{\omega}, \alpha \mid n$ denotes its restriction $\left(\alpha_{0}, \ldots, \alpha_{n-1}\right)$.

Expansion Lemma ([Jones, Th. 4], [Kom2, Th. 2], and [Kucz, p. 215]). Suppose that $S$ is Souslin- $\mathcal{H}$, i.e. of the form

$$
S=\bigcup_{\alpha \in \omega^{\omega}} \bigcap_{n=1}^{\infty} H(\alpha \mid n)
$$


with each $H(\alpha \mid n) \in \mathcal{H}$, for some family of analytic sets $\mathcal{H}$ on which $f$ is bounded. If $S$ spans $\mathbb{R}$ as a vector space over $\mathbb{Q}$ (e.g. contains a Hamel basis), then for each $n$ there are sets $H_{1}, \ldots, H_{k}$, each of the form $H(\alpha \mid n)$, such that for some integers $r_{1}, \ldots, r_{k}$,

$$
T=r_{1} H_{1}+\ldots+r_{k} H_{k}
$$

has positive measure/is non-meagre and so $T-T$ contains an interval.

Proof. For any $n \in \omega$ we have

$$
S \subseteq \bigcup_{\alpha \in \omega^{\omega}} H(\alpha \mid n)
$$

Enumerate the countable family $\left\{H(\alpha \mid n): \alpha \in \omega^{n}\right\}$ as $\left\{T_{h}: h \in \omega\right\}$. Since $S$ spans $\mathbb{R}^{d}$, we have

$$
\begin{aligned}
\mathbb{R}^{d} & =\bigcup_{h \in \omega} \bigcup_{\mathbf{k}, \mathbf{s} \in \mathbb{N}^{h}} \bigcup_{\mathbf{r} \in \mathbb{Z}^{h}}\left(\frac{r_{1}}{s_{1}} T_{k_{1}}+\ldots+\frac{r_{h}}{s_{h}} T_{k_{h}}\right) \\
& =\bigcup_{m, h \in \omega, m>0} \bigcup_{\mathbf{k} \in \mathbb{N}^{h}} \bigcup_{\mathbf{r} \in \mathbb{Z}^{h}}\left(\frac{r_{1}}{m} T_{k_{1}}+\ldots+\frac{r_{h}}{m} T_{k_{h}}\right) .
\end{aligned}
$$

As each $T_{k}$ is analytic, so too is the continuous image

$$
\frac{r_{1}}{m} T_{k_{1}}+\ldots+\frac{r_{h}}{m} T_{k_{h}}
$$

which is thus measurable/Baire. Hence, for some $h, m \in \mathbb{N}, \mathbf{k} \in \mathbb{N}^{h}$ and $\mathbf{r} \in \mathbb{Z}^{h}$, the set

$$
\frac{r_{1}}{m} T_{k_{1}}+\ldots+\frac{r_{h}}{m} T_{k_{h}}
$$

has positive measure/is non-meagre, and hence so does $r_{1} T_{k_{1}}+\ldots+r_{h} T_{k_{h}}$.

The above results will be used to prove the Main Theorem, but we may now also obtain, as a direct corollary, a reformulation and strengthening of the two theorems which motivate this paper:

Theorem JK (Theorems of Jones and Kominek). Let $f$ be additive and have either a continuous restriction or a bounded restriction $f \mid T$, where $T$ is some analytic set spanning a set of positive measure/a non-meagre set $S$. Then $f$ is continuous.

Proof. By the Analytic Dichotomy Lemma, $T$ spans $\mathbb{R}$. By the Expansion Lemma, in all cases $f$ is bounded on a set of the form $\left(r_{1} H_{1}+\ldots+r_{k} H_{k}\right)-\left(r_{1} H_{1}+\ldots+r_{k} H_{k}\right)$, which contains an interval. So by Darboux's Theorem $f$ is continuous.

In particular, taking $S=\mathbb{R}$ gives the theorems of Jones and Kominek in their original formulation.

\section{ON SPANNABILITY THEORY}

Our proof of the Analytic Automaticity Theorem relies on the Expansion Lemma of the last section and on an analysis of spanning properties of analytic sets. Here we view $\mathbb{R}$ as a vector space over $\mathbb{Q}$, and for $S \subseteq \mathbb{R}$ we denote by $\operatorname{Lin}_{\mathbb{Q}}(S)$ the linear span of $S$ regarded as a set of vectors in $\mathbb{R}$ as a space over $\mathbb{Q}$. One might expect, by analogy with classical theorems asserting that a 'large' analytic set contains a 'large' compact subset (see Rog2, Part 1 Sect. 3.5] and [Kech, Ch. III 29.E]), that perhaps for analytic $S$ the $\operatorname{span} \operatorname{Lin}_{\mathbb{Q}}(S)$ is equal to $\operatorname{Lin}_{\mathbb{Q}}(F)$ with $F$ either compact 
or $\sigma$-compact. We examine this intuition and find below that enough of this is true to enable a deduction of Jones' Theorem from Kominek's Theorem.

Our first lemma follows directly from Choquet's Capacitability Theorem [Ch4] (see especially [Del2, p. 186] and [Kech, Ch. III 30.C]). For completeness, we include the brief proof. Incidentally, the argument we employ goes back to Choquet's theorem, and indeed further, to [Dav] (see e.g. [Del1, p. 43]).

Compact Contraction Lemma. For $T$ analytic, if $T+T$ has positive Lebesgue measure, then for some compact subset $S$ of $T, S+S$ has positive measure.

Proof. We present a direct proof (see below for our original inspiration from Choquet's Theorem). As $T^{2}$ is analytic, we may write ([Rog2, p. 11]) $T^{2}=h(H)$, for some continuous $h$ and some $\mathcal{K}_{\sigma \delta}$ subset of the reals, e.g. the set $H$ of the irrationals, so that $H=\bigcap_{i} \bigcup_{j} d(i, j)$, where $d(i, j)$ are compact and, without loss of generality, the unions are each increasing: $d(i, j) \subseteq d(i, j+1)$. The map $g(x, y):=x+y$ is continuous and hence so is the composition $f=g \circ h$. Thus $T+T=f(H)$ is analytic. Suppose that $T+T$ is of positive measure. Hence, by the capacitability argument for analytic sets (see [Ch4, Si, Th. 4.2, p. 774] or [Rog1, p. 90], there referred to as an 'Increasing sets lemma'), for some compact set $A$ the set $f(A)$ has positive measure. Indeed if $|f(H)|>\eta>0$, then the set $A$ may be taken in the form $\bigcap_{i} d\left(i, j_{i}\right)$, where the indices $j_{i}$ are chosen inductively by reference to the increasing union so that $\left|f\left[H \cap \bigcap_{i<k} d\left(i, j_{i}\right)\right]\right|>\eta$, for each $k$. (Thus $A \subseteq H$ and $f(A)=\bigcap_{i} f\left[H \cap \bigcap_{i<k} d\left(i, j_{i}\right)\right]$ has positive measure; cf. [EKR $)$.

The conclusion follows as $S=h(A)$ is compact and $S+S=g(S)=f(A)$.

Note. The result may be deduced indirectly from the Choquet Capacitability Theorem by considering the capacity $I: \mathbb{R}^{2} \rightarrow \mathbb{R}$, defined by $I(X)=|g(X)|$, where, as before, $g(x, y):=x+y$ is continuous and $|$.$| denotes Lebesgue measure on \mathbb{R}$ (on this point see [Del2, Section 1.1.1, p. 186]). Indeed, the set $T^{2}$ is analytic ([Rog2, Section 2.8, p. 37-41]), so $I\left(T^{2}\right)=\sup I\left(K^{2}\right)$, where the supremum ranges over compact subsets $K$ of $T$. Actually, the Capacitability Theorem says only that $I\left(T^{2}\right)=\sup I\left(K_{2}\right)$, where the supremum ranges over compact subsets $K_{2}$ of $T^{2}$, but such a set may be embedded in $K^{2}$ where $K=\pi_{1}(K) \cup \pi_{2}(K)$, with $\pi_{i}$ being the projections onto the axes of the product space.

Corollary. For $T$ analytic and $\varepsilon_{i} \in\{ \pm 1\}$, if $\varepsilon_{1} T+\ldots+\varepsilon_{d} T$ has positive measure (measure greater than $\eta$ ) or is non-meagre, then for some compact subset $S$ of $T$, the compact set $K=\varepsilon_{1} S+\ldots+\varepsilon_{d} S$ is such that $K+K$ has positive measure (measure greater than $\eta$ ).

Proof. In the measure case the same approach may be used, based now on the continuous function $g\left(x_{1}, \ldots, x_{d}\right):=\varepsilon_{1} x_{1}+\ldots+\varepsilon_{d} x_{d}$, ensuring that $K$ is of positive measure (measure greater than $\eta$ ). In the category case, if $T^{\prime}=\varepsilon_{1} T+\ldots+\varepsilon_{d} T$ is non-meagre, then by the Steinhaus Theorem ([St] or [BGT, Cor. 1.1.3]), $T^{\prime}+$ $T^{\prime}$ contains an interval. The measure case may now be applied to $T^{\prime}$ in lieu of T. (Alternatively one may apply the Pettis-Piccard Theorem, as in the Analytic Dichotomy Lemma.)

Theorem (Compact Spanning Approximation). For $T$ analytic, if the linear span of $T$ is non-null or non-meagre, then there exists a compact subset of $T$ which spans all the reals. If $T$ is symmetric about the origin, then the compact spanning subset may be taken to be symmetric. 
Proof. If $T$ is non-null or non-meagre, then $T$ spans all the reals (by the Analytic Dichotomy Lemma). Then for some $\varepsilon_{i} \in\{ \pm 1\}, \varepsilon_{1} T+\ldots+\varepsilon_{d} T$ has positive measure/ is non-meagre. Hence for some $K$ compact, $\varepsilon_{1} K+\ldots+\varepsilon_{d} K$ has positive measure/ is non-meagre. Hence $K$ spans some and hence all reals.

Let $T$ be symmetric. If $T$ spans the reals, then so does $T_{+}=T \cap \mathbb{R}_{+}$. Choose a compact $K_{+} \subseteq T_{+}$to span the reals. Then $K:=K_{+} \cup\left(-K_{+}\right) \subseteq T$ is compact, symmetric and spans the reals.

As a corollary, we deduce the relationship between the theorems of Jones and Kominek.

Corollary. Kominek's Theorem implies Jones' Theorem.

Proof. If $T$ is an analytic spanning set, then it contains a compact spanning set $K$. If $f$ is continuous on $T$, then $f$ is bounded on the compact set $K$. By Kominek's Theorem, as $f$ is additive and bounded on a compact spanning set, $f$ is continuous.

We continue with regard to the question of whether, for $T$ analytic, there is a compact $K \subseteq T$ such that $\operatorname{Lin}_{\mathbb{Q}}(T)=\operatorname{Lin}_{\mathbb{Q}}(K)$. Evidently, the question really relates to analytic sets $T$ with $\operatorname{Lin}_{\mathbb{Q}}(T)$ (also analytic) being of measure zero or meagre (as $T$ has the Baire property), since the case where $\operatorname{Lin}_{\mathbb{Q}}(T)$ has positive measure or is non-meagre has been settled positively by the Compact Spanning Approximation Theorem. Note that if $W:=\operatorname{Lin}_{\mathbb{Q}}(T)=\operatorname{Lin}_{\mathbb{Q}}(K)$ for some compact $K$, then $\operatorname{Lin}_{\mathbb{Q}}(T)$ is $\sigma$-compact. Laczkovich Lacz showed that any proper analytic subgroup of the reals is covered by a null $\sigma$-compact set, so the $\sigma$-compact structure is not surprising. Indeed, as Roy Davies has observed (private communication), there are $\sigma$-compact proper additive subgroups $A$ of the reals which can be covered by the sums $F, F+F, F+F+F, \ldots$, for some closed set $F$ with $F=-F$, none of which contains a non-empty interval. For example $A$ may be the subgroup generated by $F$. The earliest such example is due to Sierpiński [Sierp1. We are thus led to:

Theorem (Compact Spanning Theorem). If the subspace $W$ of $\mathbb{R}$ is both $\sigma$-compact and $\mathcal{G}_{\delta}$, there exists a compact set $K$ in $W$ such that $W=\operatorname{Lin}_{\mathbb{Q}}(K)$.

Proof. This result follows from the generalized Piccard theorem (see e.g. Kom1]), since $W$ is completely metrizable ([Eng, Section 4.3]). Indeed, if $W=\bigcup_{n \in \omega} K_{n}$ with each $K_{n}$ compact, then for some $n \in \omega$ the set $K_{n}$ is non-meagre in $W$. So by Piccard's Theorem $K_{n}+K_{n}$ has non-empty interior in the topological space $W$. Let us suppose that $W$ contains the relatively open interval of points $J \cap W$. Note that for any $w \in W,(w+J) \cap W=w+(J \cap W) \subseteq W$, as $W$ is a vector space. Hence, for any $w \in J \cap W$, upon putting $I=J-w=(-a,+b)$, we have $0 \in I \cap W$ and $\mathbb{R}=\bigcup_{m \in \omega} m I$. Thus $W=\bigcup_{m \in \omega} m(I \cap W)$. We deduce that $J \cap W$ spans $W$, and the theorem follows since $K_{n}+K_{n}$ is compact.

Of course, the above theorem addresses not only the 'small case' of null and meagre subspaces of ambiguous Borel class one ([Kech, II.11.A]) but also the 'large case', e.g. the case where $W=\mathbb{R}$. Indeed, combining Theorems 6.3.3 and 6.3.4 of Jayne and Rogers in Rog2, uncountable $\mathcal{G}_{\delta}$ sets that are $\sigma$-compact are characterized up to first-level Borel isomorphism uniquely as copies of $[0,1]$. Interest in the $\mathcal{G}_{\delta}$ case here is motivated by Solecki's analytic dichotomy theorem below. 
The above result is perhaps the best one may hope for for two reasons. First of all, we refer to a further result of Laczkovich in [Lacz, where he gives an example of a null Borel subgroup $G$ of $\mathbb{R}$ with the property that for any $\sigma$-compact cover $\left\{K_{n}: n \in \omega\right\}$, there is $n$ such that $K_{n}+K_{n}$ contains an interval. By a simple modification $G$ may be assumed to be a vector subspace (e.g. $W:=\bigcup_{m \in \omega} \frac{1}{m+1} G$ is a vector subspace which is also Borel and, being null, is proper). It is not, however, $\sigma$-compact, as otherwise the representation $\bigcup_{n \in \omega} K_{n}$ would force, for some $n$, the sum $K_{n}+K_{n}$ to contain an interval, thus contradicting the fact that the subspace is null. It would be interesting to know whether there exists a $\mathcal{G}_{\delta}$ proper vector subspace of $\mathbb{R}$ which is not $\sigma$-compact. (Lavrentieff's theorem on the topological invariance of $\mathcal{G}_{\delta}$ sets - see [Eng, p. 276] - would at best permit a direct modification of Laczkovich's construction to yield a $\mathcal{G}_{\delta \sigma}$ proper vector subspace which is not $\sigma$ compact.)

Secondly, there is Solecki's analytic dichotomy theorem (reformulating and generalizing a specific instance discovered by Petruska [Pet] as follows. For $\mathcal{I}$ a family of closed sets (in any Polish space), let $\mathcal{I}_{\text {ext }}$ denote the sets covered by a countable union of sets in $\mathcal{I}$. Then, for $A$ an analytic set, either $A \in \mathcal{I}_{\text {ext }}$ or $A$ contains a $\mathcal{G}_{\delta}$ set not in $\mathcal{I}_{\text {ext }}$. See [Sol1], where a number of classical theorems asserting that a 'large' analytic set contains a 'large' compact subset are deduced, and also Sol2 for further applications of dichotomy.

We note that, by a direct appeal to Petruska's theorem in Pet, Laczkovich Lacz showed that any proper analytic subgroup of the reals is covered by a null $\sigma$-compact set.

\section{Proof of the MAIN Theorem}

Suppose that $T$ is analytic and spans $\mathbb{R}^{d}$ as a vector space over $\mathbb{Q}$ (e.g. contains a Hamel basis). Let $h \in \mathcal{F}$ be such that for every $\left\{\mathbf{x}_{n}\right\} \rightarrow \mathbf{x}_{0}$ with $\left\{\mathbf{x}_{n}\right\} \subseteq T$ (i.e. for every convergent sequence which together with its limit lies in $T$ ), we have $h \mid\left\{\mathbf{x}_{n}\right\} \in \mathcal{Q}\left(\left\{\mathbf{x}_{n}\right\}\right)$ but $h \notin \mathcal{P}$. Then $h \notin \mathcal{Q}$ (by the hypothesis that $\mathcal{Q} \Longrightarrow \mathcal{P}$ ). Since $\mathcal{Q}$ is completely sequential, there is a convergent sequence $\left\{\mathbf{u}_{m}: m \in \omega\right\}$ such that $h \mid\left\{\mathbf{u}_{m}: m \in \omega\right\} \notin \mathcal{Q}$.

Put $T_{k}=T \cap(-k, k)$. Since $T$ spans $\mathbb{R}^{d}$ as a vector space over $\mathbb{Q}$, we have by the Expansion Lemma of Section 2 that, for some $k, n \in \mathbb{N}$ and $\mathbf{r} \in \mathbb{Z}^{n}$, the set

$$
r_{1} T_{k}+\ldots+r_{n} T_{k}
$$

has positive measure. By the Compact Contraction Lemma of Section 3 there is a compact subset $S$ of $T_{k}$ such that $r_{1} S+\ldots+r_{n} S$ has positive measure.

By the Kestelman-Borwein-Ditor Theorem, for some $t$ and for $i=1, \ldots, n$, there are sequences $\left\{\mathbf{v}_{i}^{m}: m \in \mathbb{M}\right\} \subseteq S \subseteq T$ such that

$$
t+\mathbf{u}_{m}=r_{1} \mathbf{v}_{1}^{m}+\ldots+r_{n} \mathbf{v}_{n}^{m} .
$$

By the local compactness of $\mathbb{R}^{n}$, the compactness of $S$ and passage to an infinite subset $\mathbb{M}^{\prime} \subseteq \mathbb{M}$, we may assume that each sequence $\mathbf{v}_{i}=\left\{\mathbf{v}_{i}^{m}: m \in \mathbb{M}\right\}$ is convergent to a point of $S$. As each $\mathbf{v}_{i}$ is in $T$, it follows that $h \mid\left\{\mathbf{v}_{n}^{i}\right\} \in \mathcal{Q}\left(\left\{\mathbf{v}_{n}^{i}\right\}\right)$. Hence, since $\mathcal{F}$ preserves $\mathcal{Q}$ under shift and under vector addition and subtraction on the sets $\left\{\mathbf{v}_{i}^{m}: m \in \mathbb{M}\right\}$, currently assumed compact, $h \mid\left\{\mathbf{u}_{m}\right\}_{m \in \mathbb{M}} \in \mathcal{Q}\left(\left\{\mathbf{u}_{m}\right\}_{m \in \mathbb{M}}\right)$. But this contradicts $h \notin \mathcal{Q}$, since $h \mid\left\{\mathbf{u}_{m}: m \in \omega\right\} \in \mathcal{Q}$ iff $h \mid\left\{\mathbf{u}_{m}: m \in \mathbb{M}\right\} \in \mathcal{Q}$ for every infinite $\mathbb{M}$. Hence after all $h \in \mathcal{P}$. 


\section{VARIANTS: IDEALS UNDER $\mathbb{R}^{d}$-SHIFTS}

Here we point to two generalizations. The first, Theorem 1, makes explicit reference to the sequential combinatorics used in the proof of Section 4 . The second, Theorem 2, restricts attention to analytic spanning sets $T$ which are 'shiftedsymmetric'.

Definitions. Let $c\left(\mathbb{R}^{d}\right)$ denote the the sequence space of $\mathbb{R}^{d}$, i.e. the additive group of convergent sequences $\mathbf{u}=\left\{u_{n}\right\}$ of vectors in $\mathbb{R}^{d}$ (with termwise addition). A subgroup $\mathcal{G}$ that is invariant under the action of (termwise) shifts by elements of $\mathbb{R}^{d}$ will be called an $\mathbb{R}^{d}$-shift ideal (in the sequence space of $\mathbb{R}^{d}$ ); it will be called a complete $\mathbb{R}^{d}$-shift ideal if it is also closed under subsequence formation, that is:

(i) $\mathbf{u}, \mathbf{v} \in \mathcal{G}$ implies that $\mathbf{u} \pm \mathbf{v} \in \mathcal{G}$ (subgroup property);

(ii) $\mathbf{u} \in \mathcal{G}$ implies $t+\mathbf{u}=\left\{t+u_{n}\right\} \in \mathcal{G}$, for each $t$ in $\mathbb{R}^{d}$ ( $\mathbb{R}^{d}$-shift invariance); ness).

(iii) $\mathbf{u} \in \mathcal{G}$ implies that $\mathbf{u}_{\mathbb{M}}=\left\{u_{m}: m \in \mathbb{M}\right\} \in \mathcal{G}$, for every infinite $\mathbb{M}$ (complete-

Definition. We say that a sequence $\mathbf{u}=\left\{u_{n}\right\}$ is $\mathcal{Q}$-good for $h$ if

$$
h\left|\left\{u_{n}\right\} \in \mathcal{Q}\right|\left\{u_{n}\right\},
$$

and we put

$$
\mathcal{G}_{h \mathcal{Q}}=\left\{\mathbf{u}: h\left|\left\{u_{n}\right\} \in \mathcal{Q}\right|\left\{u_{n}\right\}\right\} .
$$

If $\mathcal{Q}$ is completely sequential, then $\mathbf{u}$ is $\mathcal{Q}$-good for $h$ iff every subsequence of $\mathbf{u}$ is $\mathcal{Q}$-good for $h$. One then has:

Lemma. If $\mathcal{Q}$ is completely sequential and $\mathcal{F}$ preserves $\mathcal{Q}$ under shift and under vector addition and subtraction on compacts, then $\mathcal{G}_{h \mathcal{Q}}$ for $h \in \mathcal{F}$ is a complete $\mathbb{R}^{d}$-shift ideal.

Example 3. The convergent sequences form a complete $\mathbb{R}^{d}$-shift ideal, and so do the eventually constant sequences. These give natural examples of $\mathbb{R}^{d}$-shift ideals. Further examples of interest follow Theorem 1 below.

Theorem 1 (Analytic Automaticity Theorem-combinatorial form). Suppose that

(a) functions of $\mathcal{F}$ having $\mathcal{Q}$ on $\mathbb{R}^{d}$ have $\mathcal{P}$ on $\mathbb{R}^{d}$, where $\mathcal{Q}$ is a property of functions from $\mathbb{R}^{d}$ to $\mathbb{R}$ that is completely sequential on $\mathbb{R}$;

(b) for all $h \in \mathcal{F}$, the family $\mathcal{G}_{h \mathcal{Q}}$ of $\mathcal{Q}$-good sequences is a complete $\mathbb{R}^{d}$-shift ideal (i.e. closed under vector addition and subtraction, invariant under shift, and closed under subsequence formation).

Then, for any analytic set $T$ spanning $\mathbb{R}$ as a vector space over $\mathbb{Q}$ (e.g. containing a Hamel basis), functions of $\mathcal{F}$ having $\mathcal{Q}$ on $T$ have $\mathcal{P}$ on $\mathbb{R}^{d}$.

Proof of Theorem 1. This is a rephrasing of the main theorem in the language of $\mathbb{R}^{d}$-shift ideals.

Example 4. As in Example 2 of Section 1, if $\mathcal{P}=\mathcal{C}, \mathcal{Q}=\mathcal{B}_{\text {loc }}$, and $\mathcal{F}=\mathcal{A} d d$, then $\mathcal{Q}$ is sequential and $\mathcal{F}$ preserves $\mathcal{Q}$ under vector addition and subtraction on bounded sets and also under shift and subsequence formation, so $\mathcal{G}_{h \mathcal{Q}}$ is a complete $\mathbb{R}^{d}$-shift ideal when $h \in \mathcal{F}$. Applying Theorem 1 to this example re-captures the sharpened form of Kominek's Theorem of Section 1.

For the next example we need a definition. 
Definition. Let us say that $f: T \rightarrow \mathbb{R}$ is extensibly continuous or, more simply, precompact to mean that $f$ is continuous on $T$ and if $\left\{t_{n}\right\} \subseteq T$ is Cauchy, then so is $\left\{f\left(t_{n}\right)\right\}$.

To motivate the terms, note that the definition may be rephrased in an equivalent way to require that $f$ has a continuous extension to the closure $\bar{T}$; in this case $f$ carries compacts of $\bar{T}$ to compacts. (See Bou, Section 8.5, Th. 1; cf. Eng, Section 4.3.)

If $T$ is closed the condition demands simply that $f$ be continuous on $T$. However, regarding $T$ as a test set (testing on a restriction for global continuity), one might not wish absence from $T$ of a limit point to reduce the information carried by $T$.

Example 5. For $\mathcal{P}=\mathcal{C}, \mathcal{Q}=\overline{\mathcal{C}}$ (the precompact functions) and $\mathcal{F}=\mathcal{A} d d$, we have that $\mathcal{Q}$ is sequential and that $\mathcal{F}$ preserves $\mathcal{Q}$ under vector addition and subtraction on bounded sets and also under shift, so that $\mathcal{G}_{h \mathcal{Q}}$ is a complete $\mathbb{R}^{d}$-shift ideal for $h \in \mathcal{F}$. Here again Theorem 1 applies and yields the following (new) result: a precompact additive function is continuous.

Example 6. In the case of the slowly varying functions, i.e.

$$
\mathcal{F}=\mathcal{S} v:=\left\{h:\left(\forall\left\{\mathbf{x}_{n} \rightarrow \infty\right\}\right)(\forall u) \lim _{n \rightarrow \infty}\left|h\left(u+x_{n}\right)-h\left(x_{n}\right)\right|=0\right\} .
$$

For $h$ slowly varying $(h \in \mathcal{S} v)$, let $\mathcal{G}_{h}$ be the set of convergent sequences $\mathbf{u}=\left\{u_{n}\right\}$ that are good for $h$, i.e. those such that for all $\left\{x_{n}\right\} \rightarrow \infty$,

$$
\lim _{n \rightarrow \infty}\left|h\left(u_{n}+x_{n}\right)-h\left(x_{n}\right)\right|=0 .
$$

In [BOst6] we show that $\mathcal{G}_{h}$ for $h \in \mathcal{F}$ is a complete $\mathbb{R}^{d}$-shift ideal. Granted this, Theorem 1 applies, and one deduces that the convergence above holds uniformly on compact sets. This is the Uniform Convergence Theorem (UCT) of slow (and regular) variation, for $h$ suitably regular in the sense that it is measurable or Baire. The novelty here is in the weakening of the regularity assumption; Theorem 1 yields the conclusion of the UCT for a slowly varying function $h$ provided that (lim) holds for all bounded sequences $\left\{u_{n}\right\}$ lying in an analytic set $T$ spanning $\mathbb{R}$.

Theorem 2 (Symmetric Analytic Automaticity Theorem). Suppose that

(a) functions of $\mathcal{F}$ having the property $\mathcal{Q}$ on $\mathbb{R}^{d}$ have property $\mathcal{P}$ on $\mathbb{R}^{d}$, where $\mathcal{Q}$ is a property of functions from $\mathbb{R}^{d}$ to $\mathbb{R}$ that is completely sequential on $\mathbb{R}^{d}$;

(b) $\mathcal{F}$ preserves $\mathcal{Q}$ under vector addition on compact sets and also under shift, that is:

(i) for compact sets $S$ and $T$, functions of $\mathcal{F}$ having $\mathcal{Q}$ on $S$ and $T$ have $\mathcal{Q}$ on $S+T$

(ii) functions of $\mathcal{F}$ having $\mathcal{Q}$ on any $T \subseteq \mathbb{R}^{d}$ have $\mathcal{Q}$ on $\tau+T:=\{\tau+t: t \in$ $T\}$, for any $\tau \in \mathbb{R}^{d}$.

Then, for any analytic set $T$ spanning $\mathbb{R}^{d}$ as a vector space over $\mathbb{Q}$ (e.g. containing a Hamel basis) such that, for some $\tau, S:=\tau+T$ is symmetric (i.e. $S=-S$ ), functions of $\mathcal{F}$ having $\mathcal{Q}$ on $T$ have $\mathcal{P}$ on $\mathbb{R}^{d}$.

Proof of Theorem 2. Since $S=\tau+T$ is an analytic spanning set if $T$ is, we may as well assume by (ii) that $T$ is in fact symmetric. It follows that in the Expansion Lemma all the factors $r_{i}$ may be taken positive. The result now follows from the Main Theorem, whose proof here requires only that $\mathcal{F}$ preserve $\mathcal{Q}$ under vector addition on compact sets and also under shift. 
Notes. 1. In [BOst6] we show that Theorem 2 applies also to subadditive functions and to convex functions.

2. Much of the work here carries over from the present Euclidean setting to topological groups. We develop this programme in BOst12].

3. We thank Anatole Beck for the eventual constancy part of Example 3 in this section.

\section{ACKNOWLEDGMENTS}

Dellacherie ([Del1, p. 43]) pointed out the important yet neglected work on capacities by R. O. Davies Dav in 1952 at the same time as the appearance of Choquet's three notes (Ch1]-Ch3]) in the Comptes Rendus and well before his seminal paper of 1955 Ch4. Roy Davies has been a long-standing friend, mentor and collaborator of the second author. It is a pleasure for both authors to dedicate this paper to him on the occasion of his eightieth birthday.

\section{REFERENCES}

[AD] J. Aczél and J. Dhombres, Functional equations in several variables, Encycl. Math. Appl. 31, Cambridge University Press, Cambridge, 1989. MR1004465 (90h:39001)

[Ban] S. Banach, Ueber metrische Gruppen, Studia Math. III (1931), 101-113; reprinted in Oeuvres avec des commentaires, vol. II, pp. 401-411, PWN, Warsaw, 1979. MR0563126 (81f:46001)

[BGT] N. H. Bingham, C. M. Goldie and J. L. Teugels, Regular variation, Encycl. Math. Appl., 27, Cambridge University Press, Cambridge, 1987. MR898871 (88i:26004)

[BOst] N. H. Bingham and A. J. Ostaszewski, Generic subadditive functions, Proc. Amer. Math. Soc. 136 (2008), 4257-4266. MR 2431038 (2009e:39025)

[BOst3] N. H. Bingham and A. J. Ostaszewski, Infinite combinatorics and the theorems of Steinhaus and Ostrowski, CDAM Research Report Series, LSE-CDAM-2007-15, London School of Economics, 2007.

[BOst6] N. H. Bingham and A. J. Ostaszewski, New automatic properties: subadditivity, convexity, uniformity, Aequationes Math., to appear.

[BOst9] N. H. Bingham and A. J. Ostaszewski, Infinite combinatorics in function spaces: category methods, Publ. Inst. Math. Béograd, to appear.

[BOst11] N. H. Bingham and A. J. Ostaszewski, Bitopology and measure-category duality, CDAM Research Report Series, LSE-CDAM-2007-29, London School of Economics, 2007.

[BOst12] N. H. Bingham and A. J. Ostaszewski, Normed groups: dichotomy and duality, CDAM Research Report Series, LSE-CDAM-2008-10, London School of Economics, 2008.

[Bl] A. Blass, Existence of bases implies the axiom of choice, pp. 31-33 in J. E. Baumgartner, D. A. Martin and S. Shelah (eds.), Axiomatic set theory, Contemporary Mathematics, 31, Amer. Math. Soc., Providence, RI, 1984. MR763890 (86a:04001)

[BoDi] D. Borwein and S. Z. Ditor, Translates of sequences in sets of positive measure, Canadian Mathematical Bulletin 21 (1978), 497-498. MR.523593 (80i:28018)

[Bou] N. Bourbaki, Elements of mathematics: General topology, Part 1, Addison-Wesley, Reading, MA, 1966. MR 0205210 (34:5044a)

[Ch1] G. Choquet, Capacités. Premières définitions, Comptes Rendus Acad. Sci. Paris 234 (1952), 35-37. MR0045261 (13:555b)

[Ch2] Extension et restriction d'une capacité, Comptes Rendus Acad. Sci. Paris 234 (1952), 383-385. MR0045784(13:633d)

[Ch3] Propriétés fonctionnelles des capacités alternées ou monotones. Exemples. Comptes Rendus Acad. Sci. Paris 234 (1952), 498-500. MR0047114 (13:829e)

[Ch4] Theory of capacities, Ann. Inst. Fourier (Grenoble) 5 (1953-54), 131-295. MR0080760 (18:295g)

[Dar] G. Darboux, Sur la composition des forces en statiques, Bull. des Sci. Math. 9 (1875), 281-288. 
[Dav] R. O. Davies, Subsets of finite measure in analytic sets, Nederl. Akad. Wetensch. Proc. Ser. A. 55; Indagationes Math. 14 (1952), 488-489. MR.0053184 (14:733g)

[Del1] C. Dellacherie, Capacités et processus stochastiques, Ergeb. Math., Springer-Verlag, Berlin, Heidelberg, New York, 1972. MR56:6810 MR0448504 (56:6810)

[Del2] C. Dellacherie, Un cours sur les ensembles analytiques, Part II (pp. 183-316 of [Rog2]).

[Eng] R. Engelking, General topology, Heldermann Verlag, Berlin, 1989. MR.1039321 (91c:54001)

[EKR] P. Erdős, H. Kestelman and C. A. Rogers, An intersection property of sets with positive measure, Coll. Math. 11 (1963), 75-80. MR0158961 (28:2182)

[Fal] K. J. Falconer, The geometry of fractal sets, Cambridge Tracts in Math., 85, Cambridge University Press, Cambridge, 1986. MR867284 (88d:28001)

[Jones] F. B. Jones, Measure and other properties of a Hamel basis, Bull. Amer. Math. Soc. 48 (1942), 472-481. MR0006555 (4:4e)

[Kah] J.-P. Kahane, Probabilities and Baire's theory in harmonic analysis, Twentieth century harmonic analysis - a celebration (Il Ciocco, 2000), pp. 57-72, NATO Sci. Ser. II Math. Phys. Chem., 33, Kluwer Acad. Publ., Dordrecht, 2001. MR1858779 (2002g:42002)

[Kech] A. S. Kechris, Classical descriptive set theory, Graduate Texts in Mathematics, 156, Springer, New York, 1995. MR1321597 (96e:03057)

[Kel] J. L. Kelley, General topology, Van Nostrand, New York, 1955. MR0070144 (16:1136c)

[Kes] H. Kestelman, The convergent sequences belonging to a set, J. London Math. Soc. 22 (1947), 130-136. MR0022893 (9:274j)

[Kom1] Z. Kominek, On the sum and difference of two sets in topological vector spaces, Fund. Math. 71 (1971), no. 2, 165-169. MR0293363 (45:2440)

[Kom2] Z. Kominek, On the continuity of Q-convex and additive functions, Aeq. Math. 23 (1981), 146-150. MR689027(84e:26012)

[Kucz] M. Kuczma, An introduction to the theory of functional equations and inequalities. Cauchy's functional equation and Jensen's inequality, PWN, Warsaw, 1985. MR788497 (86i:39008)

[Kur1] K. Kuratowski, Topology, Vol. I, Academic Press, New York-London; PWN, Warsaw, 1966. MR0217751 (36:840)

[Kur2] K. Kuratowski, Topology, Vol. II, Academic Press, New York-London; PWN, Warsaw, 1968. MR0259835 (41:4467)

[Lacz] M. Laczkovich, Analytic subgroups of the reals, Proc. Amer. Math. Soc. 126 (1998), 1783-1790. MR.1443837 (98g:04001)

[Ostr] A. Ostrowski, Mathematische Miszellen XIV: ̈̈ber die Funktionalgleichung der Exponentialfunktion und verwandte Funktionalgleichungen, Jahresb. Deutsch. Math. Ver. 38 (1929), 54-62 (reprinted in Collected papers of Alexander Ostrowski, Vol. 4, pp. 49-57, Birkhäuser, Basel, 1984). MR0760994 (86m:01075d)

[Pet] Gy. Petruska, On Borel sets with small cover, Real Analysis Exchange 18 (1992-93), no. 2, 330-338. MR:1228398 (95g:28003a)

[Pet1] B. J. Pettis, On continuity and openness of homomorphisms in topological groups, Ann. of Math. (2) 52 (1950), 293-308. MR0038358 (12:391d)

[Pic1] S. Piccard, Sur les ensembles de distances des ensembles de points d'un espace Euclidien, Mém. Univ. Neuchâtel, 13, Secrétariat de l'Université, Neuchâtel, 1939. MR0002901 $(2: 129 \mathrm{~d})$

[Pic2] S. Piccard, Sur des ensembles parfaites, Mém. Univ. Neuchâtel, 16, Secrétariat de l'Université, Neuchâtel, 1942. MR.0008835 (5:61n)

[Rog1] C. A. Rogers, Hausdorff measures, Cambridge University Press, London-New York, 1970. MR0281862 (43:7576)

[Rog2] C. A. Rogers, J. Jayne, C. Dellacherie, F. Topsøe, J. Hoffmann-Jørgensen, D. A. Martin, A. S. Kechris and A. H. Stone, Analytic sets, Academic Press, London, 1980.

[Sierp1] W. Sierpiński, Sur un problème de M. Lusin, Giornale di Mat. di Battaglini (3) 7 (1917), 272-277 (reprinted as [Sierp2], II, pp. 166-170).

[Sierp2] W. Sierpiński, Oeuvres choisis, Vol. I (1974), II (1975), III (1976), PWN, Warsaw. MR0414302 (54:2405), MR0414303 (54:2406) MR0414304 (54:2407)

[Si] M. Sion, Topological and measure theoretic properties of analytic sets, Proc. Amer. Math. Soc. 11, no. 5 (1960), 769-776. MR0131509 (24:A1359) 
[Sol1] S. Solecki, Covering analytic sets by families of closed sets, Journal of Symbolic Logic 59 (1994), 1022-1031. MR1295987 (95g:54033)

[Sol2] S. Solecki, Analytic ideals and their applications, Ann. Pure and Applied Logic 99 (1999), 51-72. MR1708146 (2000g:03112)

[St] H. Steinhaus, Sur les distances des points de mesure positive, Fund. Math. 1 (1920), 93-104.

[THJ] F. Topsøe and J. Hoffmann-Jørgensen, Analytic spaces and their applications, Part 3 of $[$ Rog2].

[Trau] R. Trautner, A covering principle in real analysis, Quart. J. Math. Oxford (2) 38 (1987), 127-130. MR876270 (88d:26024)

Department of Mathematics, Imperial College London, South Kensington, London SW7 2AZ, United Kingdom

E-mail address: n.bingham@ic.ac.uk

Department of Mathematics, London School of Economics, Houghton Street, LONDON WC2A 2AE, United Kingdom

E-mail address: a.j.ostaszewski@lse.ac.uk 